\title{
Ceramide-1-Phosphate, a New Mediator of Development and Survival in Retina Photoreceptors
}

\author{
Gisela E. Miranda, ${ }^{1,2}$ Carolina E. Abraban, ${ }^{1,3}$ Daniela L. Agnolazza, ${ }^{1,3}$ Luis E. Politi, ${ }^{1}$ \\ and Nora P. Rotstein ${ }^{1}$
}

Purpose. Simple sphingolipids control crucial cellular processes in several cell types. Previous work demonstrated that sphingolipids, such as ceramide, sphingosine, and sphingosine1-phosphate, are key mediators in the regulation of survival, differentiation, and proliferation of retina photoreceptors. Ceramide-1-phosphate $(\mathrm{C} 1 \mathrm{P})$ regulates growth and survival in several cell types; however, little is known concerning its functions in the retina. Whether $\mathrm{C} 1 \mathrm{P}$ also participates in controlling photoreceptor development was also explored.

Methods. Rat retina neuronal cultures were supplemented with 1 to $10 \mu \mathrm{M}$ C1P. Proliferation was determined by evaluating 5-bromo-2-deoxyuridine $(\mathrm{BrdU})$ uptake and the number of mitotic figures and differentiation by evaluating opsin and peripherin expression by immunocytochemistry and Western blot. Apoptosis was inhibited with the pan caspase inhibitor ZVADFMK and evaluated by TUNEL assay, propidium iodide/ annexin V, and DAPI labeling. Preservation of mitochondrial membrane potential was evaluated.

RESULTs. C1P enhanced BrdU uptake and increased mitosis in retinal progenitors. C1P addition advanced photoreceptor differentiation, enhancing opsin and peripherin expression and stimulating development of the apical processes in which these proteins were concentrated. In the absence of these trophic factors, photoreceptors degenerated after 4 days in vitro, and at day 6 , almost $50 \%$ of photoreceptors were apoptotic. C1P decreased photoreceptor apoptosis, reducing this percentage by half. Inhibiting caspase activity reduced photoreceptor apoptosis in the controls, but did not increase opsin expression, implying that $\mathrm{C} 1 \mathrm{P}$ has separate effects on differentiation and survival.

Conclusions. These results suggest for the first time that $\mathrm{C} 1 \mathrm{P}$ is a novel mediator that has multiple functions in photoreceptors,

From the ${ }^{1}$ Instituto de Investigaciones Bioquímicas de Bahía Blanca (INIBIBB), Universidad Nacional del Sur (UNS), Bahía Blanca, Buenos Aires, Argentina.

${ }^{2}$ Present affiliation: Instituto Multidisciplinario de Biología Celular (IMBICE), La Plata, Buenos Aires, Argentina.

${ }^{3}$ These authors contributed equally to the work presented here and should therefore be regarded as equivalent authors.

Supported by grants from FONCyT, the Argentinean National Research Council (CONICET), and the Universidad Nacional del Sur, Bahia Blanca, Argentina. NPR and LEP are independent and principal researchers from the Argentina National Research Council (CONICET), respectively, and Professors of Biological Chemistry and Cellular Biology, respectively, from the Universidad Nacional del Sur. GEM and CEA are CONICET posdoctoral fellows and DLA has a CONICET Doctoral fellowship.

Submitted for publication December 15, 2010; revised May 18, 2011; accepted June 4, 2011.

Disclosure: G.E. Miranda, None; C.E. Abrahan, None; D.L. Agnolazza, None; L.E. Politi, None; N.P. Rotstein, None

Corresponding author: Nora P. Rotstein. INIBIBB, B8000FWB Bahía Blanca, Argentina; inrotste@criba.edu.ar. initially regulating their proliferation and then promoting their survival and differentiation. (Invest Ophthalmol Vis Sci. 2011; 52:6580 - 6588) DOI:10.1167/iovs.10-7065

S phingolipids, which two decades ago were envisioned as $\checkmark$ inert membrane components, are now firmly established as bioactive molecules involved in controlling vital cellular processes. Simple sphingolipids, such as ceramide and sphingosine, induce cell cycle arrest and promote apoptosis in different cell types, whereas their phosphorylated forms, ceramide-1-phosphate (C1P) and sphingosine-1-phosphate (S1P), have the opposite functions, promoting proliferation and survival (reviewed in Refs. 1, 2). As a group, sphingolipids play crucial roles in the regulation of normal cell function and tissue homeostasis, and dysregulation of their levels and metabolism are involved in the induction and progression of an increasing number of diseases. ${ }^{3}$

The effects of C1P have been mainly demonstrated in blood or related cells, in which it regulates proliferation, migration, and survival, ${ }^{4-7}$ among other cellular processes. Increasing evidence points to a role of $\mathrm{C} 1 \mathrm{P}$ in the regulation of proliferation and apoptosis in fibroblasts and bone marrow-derived macrophages. ${ }^{2,8}$ It is also a potent proinflammatory agent, ${ }^{9}$ with a role in phagocytosis and in the regulation of macrophage chemotaxis and migration. ${ }^{7,10}$ Direct phosphorylation of ceramide by ceramide kinase (CerK) is the major identified mechanism for generating C1P. ${ }^{11}$ This enzyme was first observed in brain synaptic vesicles, and its targeting to the plasma membrane is associated with an increase in C1P levels. ${ }^{12} \mathrm{C} 1 \mathrm{P}$ is cleaved by lipid phosphate phosphatases, such as a $\mathrm{C} 1 \mathrm{P}$ phosphatase found in rat brain, ${ }^{13,14}$ or by the phosphatidic acid phosphohydrolase. ${ }^{15}$

Information on the role of $\mathrm{C} 1 \mathrm{P}$ in the nervous system is very scarce; however, the finding of enzymes of C1P metabolism, such as CerK, in synaptic vesicles ${ }^{11}$ and $\mathrm{C} 1 \mathrm{P}$ phosphatase in brain, ${ }^{13}$ the fact that $\mathrm{C} 1 \mathrm{P}$ is synthesized in granular cerebellar cells, ${ }^{16}$ and the increase in its intracellular levels in neuroblastoma cells once they initiate their differentiation, ${ }^{17}$ suggest that $\mathrm{C} 1 \mathrm{P}$ is an intracellular signal in neuronal cells as well.

The information regarding C1P's role in the eye is even scarcer. The expression of CerK has been recently reported in RPE cells, ${ }^{18}$ and that of lipid phosphate phosphatases that hydrolyze C1P has been shown in photoreceptor outer segments. ${ }^{19}$ A human CerK-like (CerKL) enzyme has been identified in the retina, and a mutation in the gene encoding it is responsible for a form of human retinitis pigmentosa. ${ }^{20,21}$ However, this enzyme was unable to phosphorylate ceramide, since no alterations in ceramide or $\mathrm{C} 1 \mathrm{P}$ levels were observed in the CerKL ${ }^{-1-}$ mouse, ${ }^{22}$ and its natural substrate and biological role have still to be uncovered. Nevertheless, these findings suggest that mutations in the enzymes controlling sphingolipid metabolism affect the balance between sphingolipid molecules with opposing functions, thus prompting the activation of neurodegenerative processes in the retina. 
Recent work from our laboratory has established the relevance of sphingolipids in controlling key processes in retina photoreceptors. ${ }^{3}$ We demonstrated that ceramide and sphingosine are mediators of photoreceptor apoptosis; their addition triggers apoptosis, and inhibition of their synthesis prevents oxidative stress-induced apoptosis of these cells. ${ }^{23,24} \mathrm{We}$ also showed that S1P actively promotes the proliferation, differentiation, and survival of photoreceptors in the vertebrate retina. $^{25}$

Searching for other sphingolipid mediators involved in regulating photoreceptor development, we investigated the role of $\mathrm{C} 1 \mathrm{P}$ in the survival, proliferation, and differentiation of rat retina photoreceptors in culture. Our results demonstrate for the first time that addition of $\mathrm{C} 1 \mathrm{P}$ at the early stages of culture was mitogenic and increased the number of photoreceptor progenitors. At later culture stages, C1P promoted the differentiation of photoreceptors and prevented apoptosis.

\section{Materials AND Methods}

Albino Wistar rats bred in our own colony were used in all the experiments. All procedures involving animals were performed in accordance with the ARVO Statement for the Use of Animals in Ophthalmic and Vision Research. Plastic 35- and 60-mm diameter culture dishes (Cellstar) were from Greiner Bio-One (Frickenhausen, Germany). Dulbecco's modified Eagle's medium (DMEM), trypsin, insulin, gentamicin, red mitochondrial stain (MitoTracKer Red CMXRos), Alexa Fluor 488-conjugated annexin V, propidium iodide (PI), 5-bromo-2deoxyuridine (BrdU), 5-bromo-2'-deoxyuridine-5' -triphosphate (BrdUTP), and terminal deoxynucleotidyl transferase (TdT) were from Invitrogen (Carlsbad, CA). Poly-L-ornithine, trypsin inhibitor, transferrin, hydrocortisone, putrescine, 4,6-diamidino-2-phenylindole (DAPI), tyramine, paraformaldehyde, and monoclonal anti-acetylated $\alpha$-tubulin antibody were from Sigma-Aldrich (St. Louis, MO). C8-ceramide-1phosphate and D-erythro (C1P) were from Biomol (Plymouth Meeting, PA). The cell-permeant pan caspase inhibitor carbobenzoxy-valyl-alanyl-aspartyl-[O-methyl]-fluoromethylketone (Z-VAD.FMK) was from Promega (Madison, WI). The monoclonal antibody against BrdU (clone G3G4) was from DSHB (developed under the auspices of the NICHD and maintained by The University of Iowa, Department of Biological Sciences, Iowa City, IA). The secondary antibody Cy2-conjugated goat anti-mouse was from Jackson ImmunoResearch (West Grove, PA). Monoclonal anti-actin antibody and the secondary antibodies used for Western blot, goat anti-mouse IgG-horseradish peroxidase (HRP) and goat anti-rabbit IgG-HRP, were from Santa Cruz Biotechnology, Inc. (Santa Cruz, CA). Fluorophore-conjugated tyramine compounds and reaction buffers were synthesized according to previous reports. ${ }^{26,27}$ The secondary antibodies biotin-conjugated goat anti-mouse and avidin-conjugated HRP were from Vector Laboratories (Burlingame, CA). Monoclonal antibodies anti-opsin (Rho4D2) and anti-peripherin (clone Per3B6) were generous gifts from Robert Molday (University of British Columbia, Vancouver, BC, Canada). Solvents were HPLC grade, and all other reagents were analytical grade.

\section{Neuronal Cultures}

Purified cultures of rat retinal neurons were prepared as previously described. ${ }^{28,29}$ Approximately $0.5 \times 10^{5}$ cells $/ \mathrm{cm}^{2}$ were seeded on 35-mm diameter dishes, pretreated with polyornithine and Schwannoma-conditioned medium, ${ }^{30}$ and then cultured in a chemically defined medium. ${ }^{29}$ Proliferation was analyzed in neuronal cultures prepared from day 0 rat retinas, in which neuroblast proliferation is very active ${ }^{31}$; these cultures were maintained for 1 day in vitro. Differentiation was evaluated in cultures obtained from retinas of 2-day-old rats; the cells were kept in culture for 6 days.

\section{Addition of C1P}

A stock solution of C1P (10 mM) was prepared in dimethylsulfoxide (DMSO); dilutions with DMEM were then prepared. C1P's effects on proliferation and differentiation were evaluated using concentrations ranging from 1 to $10 \mu \mathrm{M}$ (final concentration in the culture medium). A final 1- $\mu \mathrm{M}$ C1P concentration was chosen for subsequent experiments. The same volume of the solution used as vehicle was added to the controls.

\section{Evaluation of Neuroblast Proliferation}

To investigate C1P effect on the proliferation of photoreceptor progenitors, we supplemented neuronal cultures with C1P 1 hour after seeding the cells and proliferation was evaluated 1 day later. BrdU uptake was determined by incubating 0-day cultures with $30 \mu \mathrm{M} \mathrm{BrdU}$ (final concentration in culture) for 16 to 18 hours. Cells were fixed for at least 30 minutes, treated with $2 \mathrm{~N} \mathrm{HCl}$ for 30 minutes more for DNA denaturation, and neutralized with $0.1 \mathrm{M}$ boric acid. BrdU uptake was determined with a monoclonal antibody against BrdU. Mitotic figures were evaluated by fluorescence microscopy, after permeating cells with $0.1 \%$ Triton X-100 in phosphate-buffered saline (PBS) for 15 minutes and incubating them for 20 minutes with DAPI, a DNA marker.

\section{Immunocytochemical Methods}

Cultures were fixed for at least 1 hour with $2 \%$ paraformaldehyde (PF) in PBS at room temperature, followed by permeation with $0.1 \%$ Triton $\mathrm{X}-100$. Photoreceptors were identified by immunocytochemistry with the monoclonal antibody Rho4D2, by their morphology, and by other criteria, as previously described. ${ }^{32,33} \mathrm{Cy} 2$-conjugated goat anti-mouse was used as the secondary antibody. Tyramide signal amplification was occasionally used to improve visualization. Controls for immunocytochemistry were performed by omitting either the primary or the secondary antibody.

Cultures were then analyzed by phase-contrast and fluorescence microscopy (Eclipse E600; Nikon, Tokyo, Japan, with a C-C phasecontrast turret condenser and a Y-FL epi-fluorescence attachment), and a laser scanning confocal microscope (DMIRE2; Leica, Wetzlar, Germany) with a $\times 63$ water objective. Images were collected and processed (LCS software; Leica, and Photoshop Elements 7.0; Adobe Systems, San Jose, CA)

\section{Evaluation of Photoreceptor Differentiation}

Opsin and peripherin expression and the amount of photoreceptors with either opsin or peripherin positive-apical processes were evaluated using Rho4D2 and anti-peripherin monoclonal antibodies, respectively. ${ }^{34,35}$ Cy2-conjugated-goat anti-mouse secondary antibody and tyramide signal amplification were used for opsin and peripherin detection, respectively. For double immunostaining of acetylated $\alpha$-tubulin and peripherin, a modification of the protocol described by Uchihara et al. ${ }^{36}$ was used. The cultures were then analyzed by phasecontrast and fluorescence microscopy. Confocal microscopy was used for cultures in which double immunostaining of acetylated $\alpha$-tubulin and peripherin was performed.

\section{Protein Extraction and Western Blot Analysis}

Opsin and peripherin levels were evaluated by Western blot. The medium was removed and the cells were rinsed with PBS and collected in lysis buffer ( $3 \mathrm{mM} \mathrm{KCl}, 50 \mathrm{mM}$ Tris- $\mathrm{HCl}$ [pH 7.4], $150 \mathrm{mM} \mathrm{NaCl}, 1$ mM EDTA, $1 \%$ Tween-20, and 1\% NP-40) containing a protease inhibitor mixture and lysed in ice for 20 minutes. Proteins were quantified with a protein assay (DC; Bio-Rad, Hercules, CA), based on the Lowry assay and separated by one-dimensional SDS-PAGE. ${ }^{37}$ Proteins (either 10 or $20 \mu \mathrm{g} / \mathrm{sample}$ ) were subjected to electrophoresis on $10 \%$ SDSpolyacrylamide minigels and then transferred to PVDF membranes (Immobilon P; Millipore, Billerica, MA). The membranes were then washed in buffer with $5 \%$ nonfat milk for 1 hour at room temperature, to block nonspecific binding. Anti-opsin, anti-peripherin, and anti-actin antibodies were allowed to react with the membrane overnight at $4{ }^{\circ} \mathrm{C}$ or 2 hours at room temperature, respectively. The membranes were then thoroughly washed and incubated with HRP-conjugated goat 
anti-mouse or anti-rabbit secondary antibodies, in Tris-buffered saline Tween-20 (TBS-T) for 1 hour at room temperature. They were then visualized by using an enhanced chemiluminescence technique (ECL), according to the manufacturer's instructions. Images were obtained by scanning at 600 dpi. Densitometric quantification of the bands was performed using ImageJ software (developed by Wayne Rasband, National Institutes of Health, Bethesda, MD; available at http://rsb.info.nih. gov/ij/index.html).

\section{Evaluation of Photoreceptor Apoptosis}

Apoptosis was determined at day 6 by three different methods: terminal deoxynucleotide transferase dUTP nick-end labeling method (TUNEL) to evaluate DNA integrity, DAPI staining to assess the amount of fragmented or pyknotic nuclei, and annexin/PI labeling to evaluate phosphatidylserine translocation to the outer layer of the plasma membrane, a hallmark of apoptosis.

For TUNEL staining, the cells were fixed at day 6 with $2 \%$ PF for 15 minutes and stored in $70 \%$ ethanol for 48 hours at $-20^{\circ} \mathrm{C}$. They were then preincubated with $1 \times$ TdT buffer for 15 minutes and incubated with the TdT reaction mixture $(0.05 \mathrm{mM}$ BrdUTP and $0.3 \mathrm{U} / \mu \mathrm{L}$ TdT in TdT buffer) at $37^{\circ} \mathrm{C}$ in a humidified atmosphere for 1 hour. The reaction was stopped by a 15-minute incubation with stop buffer (300 $\mathrm{mM} \mathrm{NaCl}$ and $30 \mathrm{mM}$ sodium citrate; $\mathrm{pH} \mathrm{7.4)}$ at room temperature. Negative controls were prepared by omitting TdT. BrdU uptake was determined with an anti-BrdU monoclonal antibody, according to a standard immunocytochemical technique.

Nuclei integrity was evaluated after staining cell nuclei with DAPI; the cells were considered to be apoptotic when they showed either fragmented or condensed (pyknotic) nuclei. The percentage of apoptotic photoreceptors was determined by double labeling with DAPI and Rho4D2, to unambiguously identify cells as photoreceptors and establish the total number of these cells.

For annexin V/PI staining, the incubation medium was removed, and the dishes were washed twice with ice-cold PBS. The cells were then incubated with a 1:4 dilution of annexin $\mathrm{V}$ conjugate in annexinbinding buffer (10 $\mathrm{mM}$ HEPES, $140 \mathrm{mM} \mathrm{NaCl}$, and $2.5 \mathrm{mM} \mathrm{CaCl}_{2} ; \mathrm{pH}$ 7.4) at room temperature in the dark for 15 minutes. PI was added immediately after, and the cells were incubated for another 15 minutes in the same conditions. The cells were then washed twice in cold PBS, fixed in 1\% PF in annexin-binding buffer for 1 hour and washed in cold PBS. Labeling with annexin V, PI, or both were then analyzed by confocal microscopy.

\section{Evaluation of Mitochondrial Membrane Potential}

To assess preservation of mitochondrial membrane potential, cultures were incubated for 20 minutes before fixation with a fluorescent probe $(0.1 \mu \mathrm{g} / \mathrm{mL}$; MitoTracker Red; Invitrogen), which labels with a bright red fluorescence mitochondria retaining their membrane potential.

\section{Effect of Inhibition of Apoptosis on Opsin Expression}

To ascertain whether C1P effects on differentiation resulted from the increase in photoreceptor survival promoted by C1P, we supplemented day 1 cultures with a cell-permeant, pan-caspase inhibitor, Z-VAD.FMK, prepared in DME $(20 \mu \mathrm{M}$, final medium concentration), ${ }^{38,39}$ and 1 hour later, with or without C1P. Cells were fixed at day 6. Apoptosis was determined by evaluating nuclei integrity after DAPI staining and opsin expression was quantified by immunochemistry.

\section{Statistical Analysis}

For cytochemical studies, 10 fields per sample, randomly chosen, were analyzed in each case. Each value represents the average of at least three experiments, with three to four dishes for each condition $\pm \mathrm{SD}$. Statistical significance was determined by Student's two-tailed $t$-test.

\section{Results \\ C1P Enhanced the Proliferation of Photoreceptor Progenitors}

In neuronal cultures obtained from day 0 rat retinas, neuroblasts actively proliferate during the first 1 or 2 days, to finally differentiate mostly as photoreceptors. ${ }^{31}$ To evaluate whether C1P affected the proliferation of photoreceptor progenitors, different concentrations of $\mathrm{C} 1 \mathrm{P}$ were added to cultures prepared from day 0 rat retinas, 1 hour after seeding the cells. C1P supplementation enhanced the amount of BrdUpositive cells, compared with the controls (Figs. 1A-D). A slight increase in the amount of these cells, compared with the controls, was already observed at $1 \mu \mathrm{M} \mathrm{C} 1 \mathrm{P}$, and this increase was much higher at 5 and $10 \mu \mathrm{M} \mathrm{C1P}$. The latter concentration doubled the population of BrdU-positive cells (Fig. 1E). The amount of mitotic figures also increased with the addition of with $\mathrm{C} 1 \mathrm{P}$, from $11,200 \pm 3,400$ in the controls to $18,500 \pm 1,840$ and $26,190 \pm 4,120$ mitotic figures per dish, at 5 and $10 \mu \mathrm{M} \mathrm{C1P}$, respectively (Fig. 1F). These results suggest that $\mathrm{C} 1 \mathrm{P}$ effectively stimulated the proliferation of photoreceptor progenitors and that this effect was concentration dependent.

\section{C1P Stimulated Photoreceptor Differentiation}

We then investigated whether C1P influences photoreceptor differentiation. As we have reported, ${ }^{28,40}$ after 6 days, in vitro control cultures lacking photoreceptor trophic factors showed a few photoreceptors expressing opsin; $1 \mu \mathrm{M} \mathrm{C1P}$ sharply increased the amount of opsin-positive photoreceptors per dish, from approximately 30,000 to more than 50,000 (Fig. 2A). Higher C1P concentrations also increased, although not significantly, the number of photoreceptors expressing opsin. Western blot assays confirmed this increase; higher levels of opsin were clearly evident in C1P-supplemented cultures, compared with those observed in the controls, after 6 days in vitro (Fig. 2B).

We then analyzed the expression of peripherin, a structural protein found in the rims of outer segment discs that allows the correct folding and maintenance of these structures. Addition of C1P promoted peripherin expression; peripherin-positive photoreceptors increased approximately $30 \%$ in $1 \mu \mathrm{M} \mathrm{C} 1 \mathrm{P}$ supplemented cultures compared with the controls (Fig. 3A). This increase in peripherin levels was confirmed by Western blot analysis (Fig. 3B).

Next, we investigated C1P's effect on the development of apical processes. As previously reported, ${ }^{28,40}$ most photoreceptors in control cultures failed to develop apical processes. A small number of photoreceptors had acetylated $\alpha$-tubulinstained cilia (Figs. 4A, 4C, arrowheads), and peripherin immunoreactivity was observed only occasionally (Figs. 4B, 4C, arrows; Fig. 4G, open arrow), usually localized in the cilia (Fig. 4C, merged image). Opsin expression was diffusely distributed throughout their cell bodies, neurites, and, when present, in cilia (Fig. 4I, open arrow). After the addition of C1P, many photoreceptors developed prominent apical processes, intensely stained with peripherin (Figs. $4 \mathrm{E}, 4 \mathrm{~F}, 4 \mathrm{H}$, arrows) and opsin (Fig. 4J, arrow), protruding from $\alpha$-tubulin-labeled cilia (Fig. 4D, arrowhead). More than 15\% of opsin-positive photoreceptors had apical processes in control cultures (Fig. 5A); addition of $\mathrm{C} 1 \mathrm{P}$ doubled the percentage of photoreceptors with apical processes at all concentrations assayed. The effect of C1P on advancing photoreceptor differentiation was evident when we analyzed the distribution of peripherin-positive photoreceptors that had developed cilia or cilia plus apical processes. Among the few photoreceptors expressing peripherin in control cultures, most (almost 75\%) had only cilia, and only 
Ctl
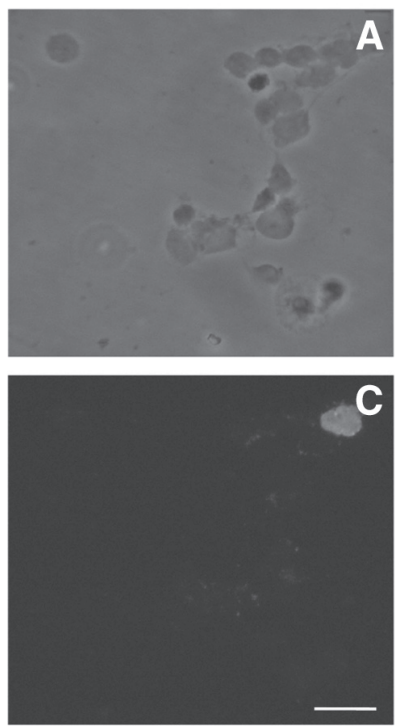

C

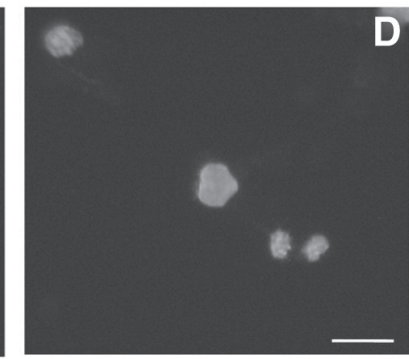

$\mathbf{E}$

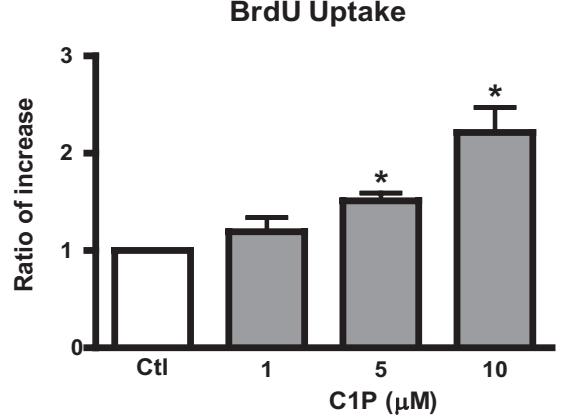

$\mathbf{F}$

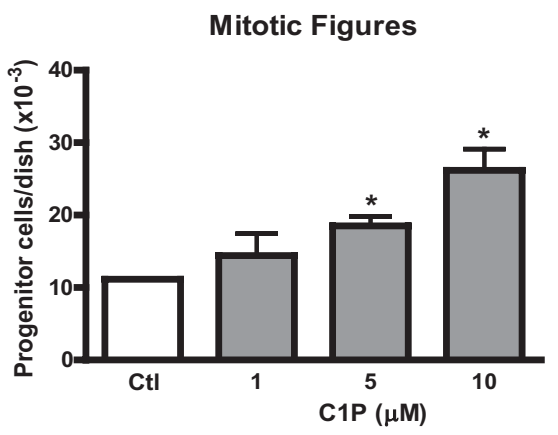

FigURE 1. C1P increased BrdU incorporation and mitosis in photoreceptor progenitors. Neuronal cultures prepared from retinas of day 0 rat pups were supplemented with 1,5 , and $10 \mu \mathrm{M} \mathrm{C1P}$ or with vehicle (Ctl) 1 hour after seeding the cells; 4 hours later, they were incubated with BrdU for 18 hours and then fixed. Phase-contrast $(\mathbf{A}, \mathbf{B})$ and fluorescence $(\mathbf{C}, \mathbf{D})$ micrographs of control $(\mathrm{Ctl})(\mathbf{A}, \mathbf{C})$ and $\mathrm{C} 1 \mathrm{P}-$ supplemented (B, D) cultures showing BrdU uptake (C, D). Ratio of increase of BrdU-positive neuronal progenitors, compared with the controls (E) and number of progenitor cells per dish showing mitotic figures $(\mathbf{F})$. *Statistically significant differences, compared with the controls $(P \leq 0.05)$. Scale bar, $10 \mu \mathrm{m}$.

$\sim 25 \%$ of them developed apical processes. In contrast, in C1P-supplemented cultures, $\sim 70 \%$ of peripherin-positive photoreceptors developed apical processes and cilia (Fig. 5B), and only $\sim 30 \%$ stopped their differentiation after developing cilia.

These results allow us to propose that $\mathrm{C} 1 \mathrm{P}$ stimulates the synthesis of proteins characteristic of mature photoreceptors, such as opsin and peripherin, and also promotes the formation of apical processes, in which these proteins are localized.

\section{C1P Promoted Photoreceptor Survival In Vitro}

In cultures lacking trophic factors for photoreceptors, these neurons develop for 2 to 3 days and then start degenerating through an apoptotic pathway. ${ }^{28}$ Previous work from our laboratory has shown that supplementation with DHA and S1P reduces the apoptosis. ${ }^{25,41,42}$ In the present study, we investigated whether C1P had a similar protective effect on photoreceptors. Apoptosis of neuronal cells is associated with the presence of many annexin V/PI-labeled neurons. ${ }^{24,43}$ By day 6 , photoreceptor degeneration due to the lack of trophic factors has already started, ${ }^{44}$ as evidenced by the amount of annexin V/PI (Figs. 6A-D, arrows) and TUNEL-labeled cells (Figs. 6I, 6J). A considerable reduction in annexin V/PI (Figs. 6E-G, arrow in $\mathrm{H}$ ) and TUNEL-labeled cells (Figs. 6K, 6L) was evident in C1Psupplemented cultures. Evaluation of nuclei integrity established that $\sim 45 \%$ of photoreceptors were apoptotic in control cultures after 6 days in culture; addition of C1P decreased this percentage to less than $25 \%$ (Fig. 6M). This result suggests that C1P promotes the survival of photoreceptors, reducing their apoptosis during development in vitro.

A

Opsin Expression

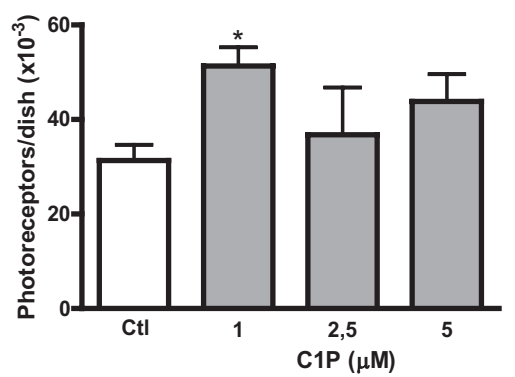

B
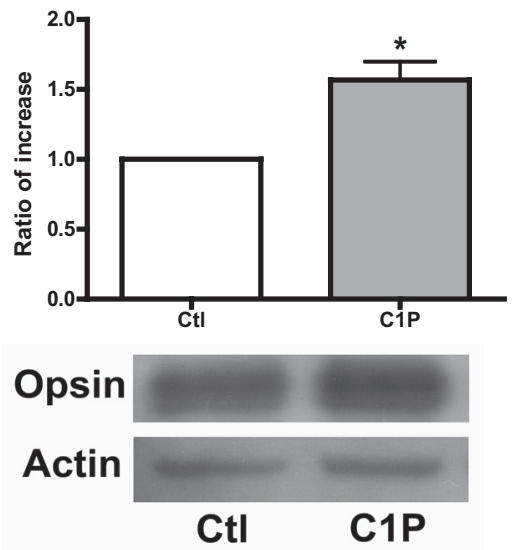

Figure 2. C1P increased opsin levels. Neuronal cultures prepared from retinas obtained from day 2 rats were supplemented at day 1 with $1,2.5$, or $5 \mu \mathrm{M} \mathrm{C1P}$ or with vehicle (Ctl) and then fixed or lysed at day 6 in vitro for immunocytochemistry or Western blot analysis, respectively, to evaluate opsin expression. Effect of C1P concentration on the number of photoreceptors expressing opsin per dish (A); each value represents the average of at least three experiments, with three to four dishes for each condition $\pm \mathrm{SD}$; at least 10 fields, randomly chosen, were counted for each dish. Western blots and their densitometric analyses show opsin expression in day 6 cultures without (Ctl) or with $1 \mu \mathrm{M}$ C1P supplementation (B); Western blot analysis and densitometric analyses are representative of three independent experiments with similar results. "Statistically significant differences compared to controls $(P \leq 0.05)$. 
A

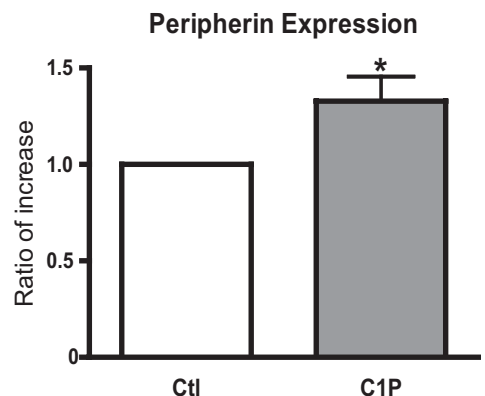

B
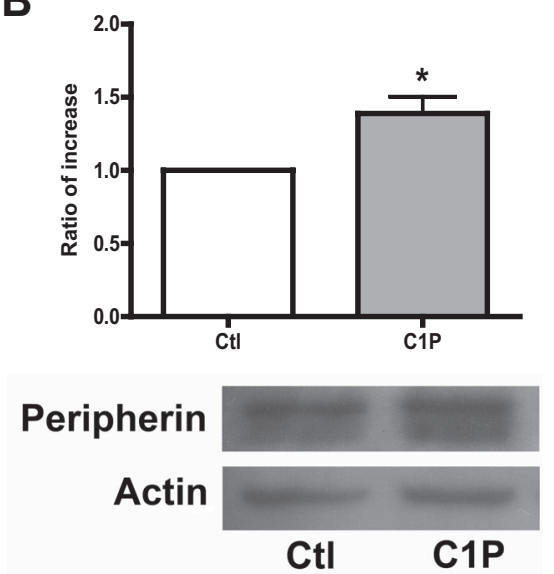

Figure 3. C1P promoted peripherin expression. Neuronal cultures prepared from retinas from 2-day-old rats, with or without $1 \mu \mathrm{M} \mathrm{C1P}$ supplementation and fixed or lysed at day 6 , for immunocytochemistry or Western blot analysis, respectively, to evaluate peripherin expression. Ratio of increase in the number of peripherin-expressing photoreceptors with respect to the controls $(\mathbf{A})$; each value represents the average of at least three experiments, with three to four dishes for each condition $\pm \mathrm{SD}$; at least 10 fields, randomly chosen, were counted for each dish. Western blots and their densitometric analyses showing changes in peripherin levels (B); the nonglycosylated (bottom band) and the glycosylated (top band) forms of peripherin are shown. Western blot analysis and densitometric analyses are representative of three independent experiments with similar results. *Statistically significant differences compared to the controls $(P \leq 0.05)$.

Apoptosis of photoreceptors due to the lack of trophic factors involves the loss of their mitochondrial membrane potential $^{43}$; this was clearly evident in control, 6-day neuronal cultures (Figs. 6N, 6O, arrowheads). C1P preserved mitochondrial potential; many photoreceptors with brilliantly labeled mitochondria were observed in C1P-supplemented cultures (Figs. 6P, 6Q, thin arrows). Although only 35\% of photoreceptors preserved mitochondrial potential in the controls, this percentage increased to almost $60 \%$ after C1P addition (Fig. 6R).

\section{Distinguishing between C1P Survival and Differentiation Effects}

The described results provided evidence that C1P promotes both survival and photoreceptor differentiation. To ascertain whether increased differentiation resulted from increased survival or whether they were separate effects, we inhibited caspase activation, which we have previously shown to participate in photoreceptor apoptosis in culture. ${ }^{42}$ Addition of ZVAD-FMK, a pan-caspase inhibitor, to control cultures significantly decreased photoreceptor apoptosis by day 6 in vitro, to levels similar to those found in C1P-supplemented cultures (Fig. 7A). Despite the reduction in apoptosis, opsin-expressing photoreceptors remained at percentages similar to those found in cultures lacking the caspase inhibitor (Fig. 7B). Of note, the presence of this inhibitor did not affect photoreceptor survival and differentiation in C1P-supplemented cultures.

\section{Discussion}

Our recent work has established that simple sphingolipids such as ceramide, sphingosine, and S1P are crucial players in the development and decision between death or survival of retina photoreceptors. In this work, we showed for the first time a role of $\mathrm{C} 1 \mathrm{P}$ in the development of photoreceptor neurons in vitro. Its addition initially stimulated the proliferation of neuroblasts in culture and then advanced the differentiation of photoreceptors, promoting the formation of apical processes and prompting an increase in the levels of constitutive proteins of photoreceptor outer segments, such as opsin and peripherin. It also decreased the apoptosis of photoreceptors during their early development in culture. The present results suggest that C1P may be a further bioactive sphingolipid involved in the regulation of the proliferation, differentiation, and survival of photoreceptors.

Although C1P was identified over two decades ago, its roles started to be uncovered during the past decade; recent work has established it as a key regulator of cell growth and survival
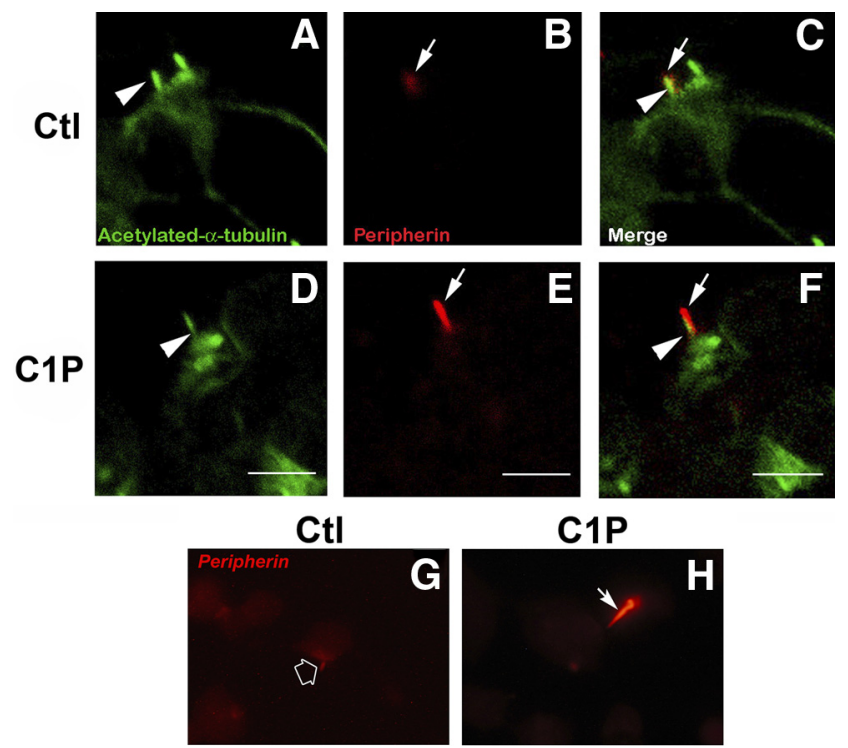

C1P

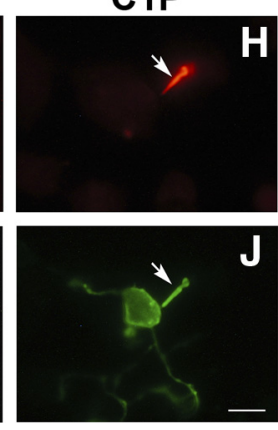

Figure 4. C1P stimulated opsin and peripherin localization in photoreceptors apical processes. Neuronal cultures prepared from retinas obtained from 2-day-old rats without (A-C, G, I) or with $1 \mu \mathrm{M} \mathrm{C1P}$ (D-F, H, J) added at day 1 were fixed at day 6. Confocal (A-F) and fluorescence photomicrographs show labeling with $\alpha$-acetylated tubu$\operatorname{lin}(\mathbf{A}, \mathbf{D})$, peripherin ( $\mathbf{B}, \mathbf{E}, \mathbf{G}, \mathbf{H}$, arrows $)$, merged labeling $(\mathbf{C}, \mathbf{F})$ and opsin (I, J). Note that in control cultures $\alpha$-acetylated tubulin-stained cilia (A, C, arrowbeads) colocalized with peripherin labeling (C, arrowbead and arrow), which showed only weak staining (G, open arrow); opsin staining was evident in cell body and cilium (I, open arrow). In C1P-supplemented cultures, photoreceptors had prominent apical processes that protruded from $\alpha$-acetylated tubulin-labeled cilia (D, F, arrowbeads) and showed an intense peripherin (E, H, arrows) and opsin labeling (J, arrow). Scale bar, $5 \mu \mathrm{m}$. 
A

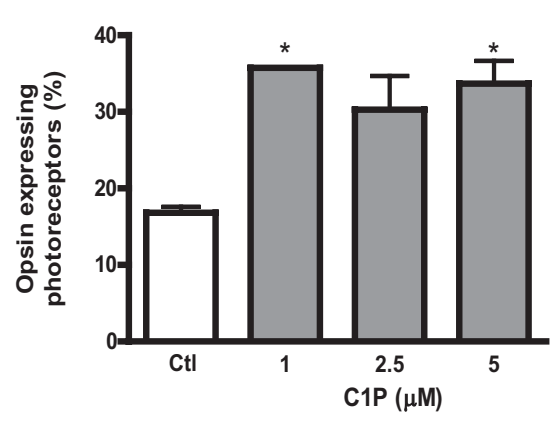

B

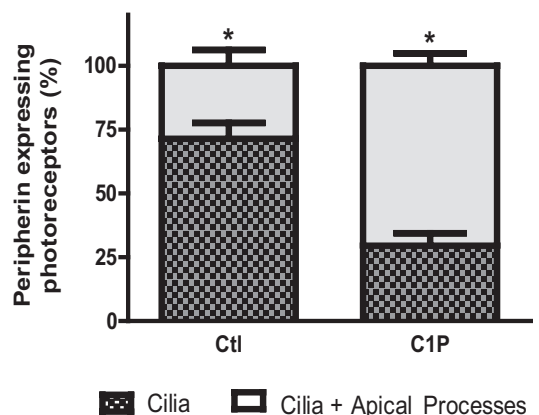

FIGURE 5. C1P promoted the development of apical processes. Neuronal cultures supplemented at day 1 without $(\mathrm{Ctl})$ or with 1 , 5, or 10 $\mu \mathrm{M}$ C1P were incubated for 5 days and then immunostained to detect opsin and peripherin. The effect of the concentration of C1P on the formation of apical processes in opsin-positive photoreceptors (A) was studied. The percentage of peripherin-positive photoreceptors developing only cilia or cilia plus apical processes (B) without (Ctl) or with C1P were also determined. "Statistically significant differences compared to the controls $(P \leq 0.05)$.

in macrophages and fibroblasts. ${ }^{8}$ Its exogenous addition induces several responses, such as cell survival, DNA synthesis, and increase in $\mathrm{Ca}^{2+}$ levels. ${ }^{2,45} \mathrm{C} 1 \mathrm{P}$ functions in the retina are virtually unknown. In Drosophila, a mutation in CerK leads to failure in the phototransduction pathway and degeneration in photoreceptors. ${ }^{46}$ Particularly intriguing is the identification in the retina of a CerKL enzyme, homolog to CerK, since an autosomal recessive form of human retinitis pigmentosa is due to its mutation ${ }^{20,21}$; this is the first direct evidence of a relationship between sphingolipid metabolism and retinal degeneration. Though as stated above, CERKL does not seem to regulate ceramide nor C1P levels, ${ }^{22}$ its overexpression protects cultured cells from oxidative-stress-induced apoptosis.

\section{C1P as a Promoter of Proliferation and Differentiation of Photoreceptors}

In mice, genesis of rod photoreceptors starts at embryonic day 13 , peaks at birth, and many progenitor cells remain mitotically active postnatally until as late as postnatal days 3 to $5 .{ }^{47,48} \mathrm{We}$ have shown that cultures obtained from day 0 rat retinas show many mitotically active neuroblasts, which differentiate mainly as photoreceptors. ${ }^{25,31}$ Uncovering molecular signals able to regulate the proliferation of progenitor cells and instruct their later differentiation as photoreceptors has become increasingly relevant, since the finding of stem cells in the retina, which may provide a therapeutic alternative to replace lost neurons in retinal degenerations. The present results show that exogenous addition of C1P to postnatal day $(\mathrm{PN}) 0$ neuronal cultures had a mitogenic effect, promoting the proliferation of photo- receptor progenitors. As stated above, $\mathrm{C} 1 \mathrm{P}$ has been shown to enhance proliferation in fibroblasts and macrophages, although at a much higher concentration. ${ }^{5,49}$ Recent work has uncovered the intracellular pathways activated by C1P in macrophages to promote cell division, which include the phosphatidylinosititol-3-kinase (PI3K)/protein kinase B (or Akt), the MAPK (mitogen protein kinase)/extracellularly regulated (ERK) 1-2 and c-Jun N-terminal kinase (JNK) pathways and the translocation of PKC (protein kinase C)- $\alpha$ from cytosol to the cell membrane. ${ }^{5,50}$ Although further research is needed to establish whether C1P turns on similar pathways in retinal neuroblasts, our results clearly support $\mathrm{C} 1 \mathrm{P}$ as one of the molecular cues that activate the cell cycle in photoreceptor progenitors.

Development of photoreceptors is a multistep process; once they undergo their final mitosis, they gradually advance in their differentiation, requiring several days to express specific proteins and acquire their characteristic morphology. They initially develop a connecting cilium and then start expressing opsin and assembling and accumulating opsin-enriched membranes at the tip of the cilium. These membranes will eventually form the outer segment discs, where all the phototransduction machinery is located. In vitro, the differentiation of photoreceptors seems to be arrested in the absence of trophic factors, with photoreceptor cells resembling their immature in vivo counterparts. ${ }^{44}$ Exogenously added C1P promoted photoreceptor differentiation, and it increased the levels of opsin and peripherin, two photoreceptor-specific proteins that are essential components of photoreceptor outer segments. C1P also stimulated the formation of apical processes that resemble rudimentary outer segments and promoted the localization of opsin and peripherin in these processes. It is noteworthy that CERK overexpression has been shown to inhibit differentiation induced by all-trans retinoic acid in a neuroblastoma cell line, ${ }^{51}$ suggesting that $\mathrm{C} 1 \mathrm{P}$ effect on differentiation depends on the cell type.

We have shown that S1P and glial-derived neurotrophic factor (GDNF), a photoreceptor trophic factor stimulate the cell cycle in photoreceptor progenitors. Moreover, GDNF upregulates the expression of sphingosine kinase-1, the enzyme responsible for $\mathrm{S} 1 \mathrm{P}$ synthesis, thus increasing the levels of $\mathrm{S} 1 \mathrm{P}$, which signals GDNF mitogenic effect. ${ }^{25}$ Likewise, we have demonstrated that S1P and docosahexaenoic acid (DHA) allow photoreceptor differentiation to proceed, ${ }^{25,28,40,52}$ as C1P did. As is the case with GDNF, S1P is a mediator of the effects of DHA, which also promotes S1P synthesis by upregulating sphingosine kinase-1 expression. ${ }^{25}$ Information implicating trophic factors in the regulation of C1P levels is still scarce. IL1- $\beta$ increases C1P levels in a pulmonary adenocarcinoma cell line. ${ }^{53}$ TNF- $\alpha$ stimulates CerK activity in HL-60 cells, ${ }^{54}$ whereas downregulation of CerK reduces growth, promotes apoptosis, and blocks epithelial growth factor-induced proliferation in lung adenocarcinoma cells. ${ }^{55}$ This leads us to propose that the synthesis of $\mathrm{C} 1 \mathrm{P}$ in photoreceptors might be modulated by trophic factors such as GDNF and DHA, to promote the proliferation and later differentiation of photoreceptor progenitors; however, establishing whether $\mathrm{C} 1 \mathrm{P}$ acts as a second messenger for photoreceptor trophic factors necessitates further research.

\section{C1P as an Antiapoptotic Mediator in Photoreceptors}

C1P has been shown to promote cell survival, acting as a potent inhibitor of apoptosis in macrophages and lung epithelial cells. ${ }^{6,8,56,57}$ Our results are the first to show a protective effect of $\mathrm{C} 1 \mathrm{P}$ on neurons, promoting the survival of photoreceptors during their development in vitro. Previous work has 

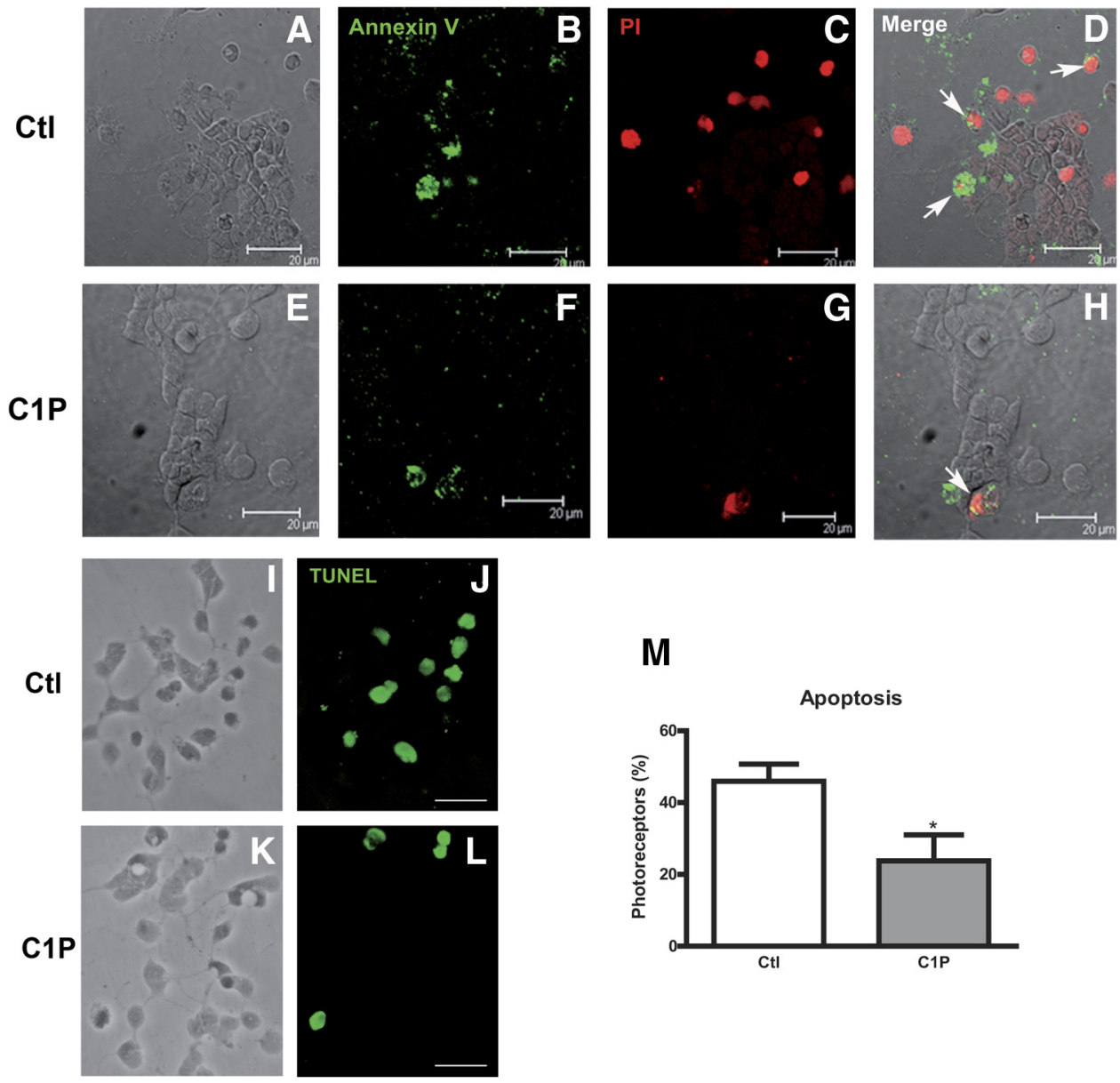

M

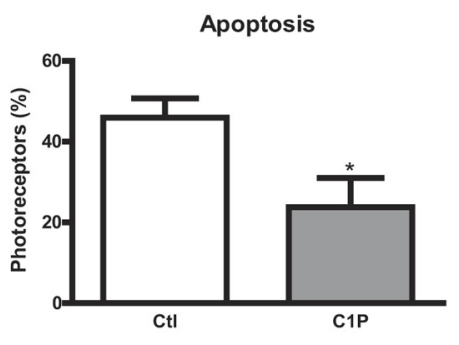

$\mathbf{R}$

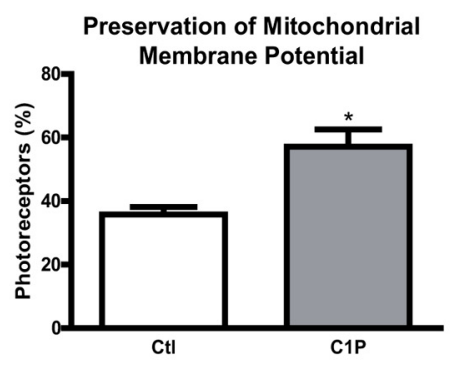

Figure 6. C1P prevented photoreceptor apoptosis during development in vitro. Cultures supplemented with vehicle (A-D, I, J, N, O) or with $1 \mu \mathrm{M} \mathrm{C1P}(\mathbf{E}-\mathbf{H}, \mathbf{K}, \mathbf{L}, \mathbf{P}, \mathbf{Q})$ at day 1 , were fixed at day 6 . Nomarsky (A, E), phase-contrast (I, K), confocal fluorescence $(\mathbf{B}, \mathbf{C}, \mathbf{F}, \mathbf{G})$, fluorescence $(\mathbf{J}, \mathbf{L}, \mathbf{N}, \mathbf{P})$, and merged Nomarsky+fluorescence (D, H, O, Q) micrographs show annexin $\mathrm{V}$ (B, $\mathbf{F}$, green), PI (C, G, red fluorescence), annexin V/PI (D, H), TUNEL $(\mathbf{J}, \mathbf{L})$, and red mitochondrial tracer (MitoTracker; Invitrogen) (N, O, P, Q) labeling. The percentage of apoptotic photoreceptors (M) was determined by quantifying the number of pyknotic, or fragmented, nuclei with DAPI. The percentage of photoreceptors preserving their mitochondrial membrane potential (R) was evaluated using the mitochondrial tracer. Note that the amount of cells double labeled with annexin $\mathrm{V}$ and PI in control cultures $(\mathbf{B}, \mathbf{C}$, arrows in D) and TUNEL-positive cells (J) were markedly reduced in C1P-supplemented cultures $(\mathbf{F}, \mathbf{G}$, arrows in $\mathbf{H}$; L). Mitochondrial depolarization was evident in many photoreceptors in the controls ( $\mathbf{N}, \mathbf{O}$, arrowheads); addition of C1P preserved photoreceptor mitochondrial potential $(\mathbf{P}, \mathbf{Q}$, thin arrows). *Statistically significant difference compared with the control $(P<0.05)$. Scale bar, $20 \mu \mathrm{m}$. shown that an increase in ceramide levels triggers apoptosis of photoreceptors in different situations of cell stress, ${ }^{23}$ and this death involves the loss of mitochondrial potential. ${ }^{23} \mathrm{C} 1 \mathrm{P}$ protection of photoreceptors involves the preservation of this potential, suggesting that it activates pathways that are upstream mitochondrial depolarization. C1P has been shown to prevent apoptosis in macrophages by blocking the increase in ceramide levels that triggers apoptosis, by inhibiting either acid sphingomyelinase or de novo synthesis of ceramide. ${ }^{6,57}$ C1P protection is similar to that exerted by S1P and DHA, which prevent photoreceptor apoptosis at early stages of in vitro culture. ${ }^{25,28,42,44}$ Inhibiting ceramide accumulation prevents apoptosis in retina photoreceptors in vitro, and DHA protective effects involve the upregulation of the expression of enzymes that decrease ceramide levels. ${ }^{23}$ Similarly, photoreceptor trophic factors may upregulate CerK expression as an additional way of avoiding a dangerous increase in intracellular ceramide content.

In our study, C1P stimulated photoreceptor differentiation, increasing opsin and peripherin expression, and concurrently enhanced photoreceptor survival, decreasing apoptosis. These may be separate effects, in a sequential pathway, or increased differentiation might simply be a consequence of a larger population of surviving photoreceptors being able to advance their differentiation. Since we have established that at least caspase- 3 is activated during photoreceptor apoptosis in vitro, ${ }^{42}$ we used a pan-caspase inhibitor to distinguish between these two alternative explanations. Although this inhibitor con- 
A

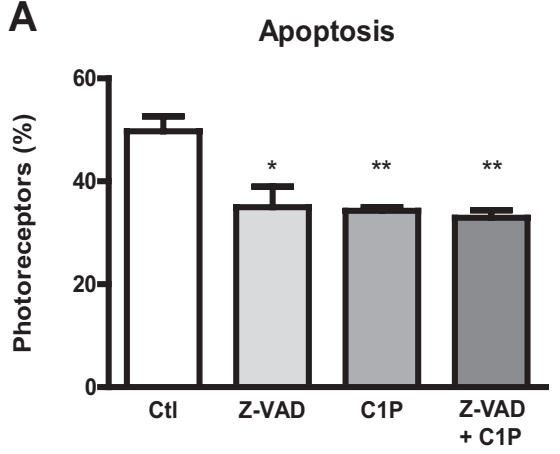

B

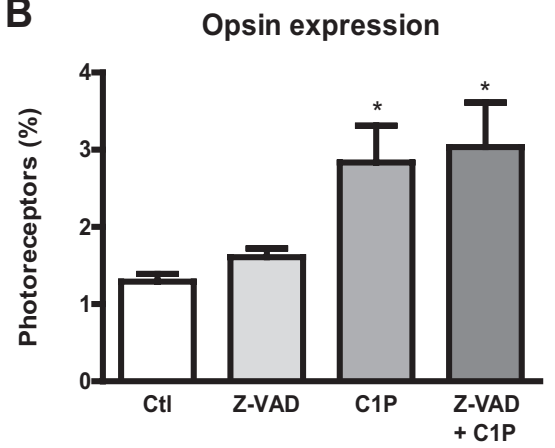

FiguRE 7. C1P had separate effects on survival and differentiation Day 1 cultures were treated with the pan caspase inhibitor, Z-VAD.FMK (20 $\mu \mathrm{M}$, final medium concentration), and vehicle (Ctl) or C1P was added 1 hour later. At day 6, apoptosis was determined by evaluating nuclear integrity after DAPI staining and opsin expression was evaluated by cytochemistry. "Statistically significant difference compared with the control $(P<0.05)$; ${ }^{* *}$ Statistically significant difference compared with the control $(P<0.01)$.

siderably reduced photoreceptor apoptosis in cultures lacking C1P, no simultaneous increase in opsin expression accompanied this reduction. Remarkably, the amount of apoptotic photoreceptors in C1P-supplemented cultures was the same in cultures, with or without the caspase inhibitor. These results imply that C1P effects on photoreceptor differentiation do not result from an increased survival of these cells, but are separate effects. They also suggest that $\mathrm{C} 1 \mathrm{P}$ inhibits caspase activation, probably through its prevention of mitochondrial membrane permeabilization, as part of its antiapoptotic effect.

In conclusion, these results are the first evidence that $\mathrm{C} 1 \mathrm{P}$ acts as an intracellular signal in photoreceptors, promoting the proliferation of photoreceptor progenitors during early development in vitro, and later on, their differentiation and survival. These multiple effects of $\mathrm{C} 1 \mathrm{P}$, together with those previously established for ceramide, sphingosine, and S1P underscore the relevance of sphingolipids as key mediators in the regulation of development and survival in photoreceptors.

\section{References}

1. Gómez-Muñoz A. Modulation of cell signalling by ceramides Biochim Biophys Acta. 1998;1391:92-109.

2. Gómez-Muñoz A. Ceramide-1-phosphate: a novel regulator of cell activation. FEBS Lett. 2004;562:5-10.

3. Rotstein NP, Miranda GE, Abrahan CE, German OL. Regulating survival and development in the retina: key roles for simple sphingolipids. J Lipid Res. 2010;51:1247-1262.

4. Gangoiti P, Granado MH, Arana L, Ouro A, Gómez-Muñoz A. Involvement of nitric oxide in the promotion of cell survival by ceramide 1-phosphate. FEBS Lett. 2008;582:2263-2269.

5. Gangoiti P, Granado MH, Wang SW, Kong JY, Steinbrecher UP, Gómez-Muñoz A. Ceramide 1-phosphate stimulates macrophage proliferation through activation of the PI3-kinase/PKB, JNK and ERK1/2 pathways. Cell Signal. 2008;20:726-736.

6. Granado MH, Gangoiti P, Ouro A, Arana L, Gómez-Muñoz A. Ceramide 1-phosphate inhibits serine palmitoyltransferase and blocks apoptosis in alveolar macrophages. Biochim Biophys Acta. 2009;1791:263-272.

7. Granado MH, Gangoiti P, Ouro A, et al. Ceramide 1-phosphate (C1P) promotes cell migration involvement of a specific C1P receptor. Cell Signal. 2009;21:405-412.

8. Gómez-Muñoz A. Ceramide 1-phosphate/ceramide, a switch between life and death. Biochim Biophys Acta. 2006;1758:20492056.

9. Chalfant CE, Spiegel S. Sphingosine 1-phosphate and ceramide 1-phosphate: expanding roles in cell signaling. J Cell Sci. 2005;118: 4605-4612.

10. Arana L, Gangoiti P, Ouro A, Trueba M, Gómez-Muñoz A. Ceramide and ceramide 1-phosphate in health and disease. Lipids Health Dis. 2010;9:15.

11. Bajjalieh SM, Martin TF, Floor E. Synaptic vesicle ceramide kinase: a calcium-stimulated lipid kinase that co-purifies with brain synaptic vesicles. J Biol Chem. 1989;264:14354-14360.

12. Kim TJ, Mitsutake S, Igarashi $Y$. The interaction between the pleckstrin homology domain of ceramide kinase and phosphatidylinositol 4,5-bisphosphate regulates the plasma membrane targeting and ceramide 1-phosphate levels. Biochem Biophys Res Commun. 2006;342:611-617.

13. Shinghal R, Scheller RH, Bajjalieh SM. Ceramide 1-phosphate phosphatase activity in brain. J Neurochem. 1993;61:2279-2285.

14. Boudker O, Futerman AH. Detection and characterization of ceramide-1-phosphate phosphatase activity in rat liver plasma membrane. J Biol Chem. 1993;268:22150-22155.

15. Waggoner DW, Gómez-Muñoz A, Dewald J, Brindley DN. Phosphatidate phosphohydrolase catalyzes the hydrolysis of ceramide 1-phosphate, lysophosphatidate, and sphingosine 1-phosphate. J Biol Chem. 1996;271:16506-16509.

16. Riboni L, Bassi R, Anelli V, Viani P. Metabolic formation of ceramide-1-phosphate in cerebellar granule cells: evidence for the phosphorylation of ceramide by different metabolic pathways. Neurochem Res. 2002;27:711-716.

17. Condorelli F, Sortino MA, Stella AM, Canonico PL. Relative contribution of different receptor subtypes in the response of neuroblastoma cells to tumor necrosis factor-alpha. J Neurochem. 2000;75: 1172-1179.

18. Zhu D, Sreekumar PG, Hinton DR, Kannan R. Expression and regulation of enzymes in the ceramide metabolic pathway in human retinal pigment epithelial cells and their relevance to retinal degeneration. Vision Res. 2010;50:643-651.

19. Pasquare SJ, Salvador GA, Giusto NM. Involvement of lysophosphatidic acid, sphingosine 1-phosphate and ceramide 1-phosphate in the metabolization of phosphatidic acid by lipid phosphate phosphatases in bovine rod outer segments. Neurochem Res. 2008;33:1205-1215

20. Tuson M, Marfany G, Gonzalez-Duarte R. Mutation of CERKL, a novel human ceramide kinase gene, causes autosomal recessive retinitis pigmentosa (RP26). Am J Hum Genet. 2004;74:128-138.

21. Auslender N, Sharon D, Abbasi AH, Garzozi HJ, Banin E, Ben-Yosef T. A common founder mutation of CERKL underlies autosomal recessive retinal degeneration with early macular involvement among Yemenite Jews. Invest Ophthalmol Vis Sci. 2007;48:54315438.

22. Graf C, Niwa S, Muller M, Kinzel B, Bornancin F. Wild-type levels of ceramide and ceramide-1-phosphate in the retina of ceramide kinase-like-deficient mice. Biochem Biophys Res Commun. 2008; 373:159-163.

23. German OL, Miranda GE, Abrahan CE, Rotstein NP. Ceramide is a mediator of apoptosis in retina photoreceptors. Invest Ophthalmol Vis Sci. 2006;47:1658-1668.

24. Abrahan CE, Miranda GE, Agnolazza DL, Politi LE, Rotstein NP. Synthesis of sphingosine is essential for oxidative stress-induced apoptosis of photoreceptors. Invest Ophthalmol Vis Sci. 2010;51: 1171-1180.

25. Miranda GE, Abrahan CE, Politi LE, Rotstein NP. Sphingosine-1phosphate is a key regulator of proliferation and differentiation in 
retina photoreceptors. Invest Ophthalmol Vis Sci. 2009;50:44164428.

26. Bobrow MN, Shaughnessy KJ, Litt GJ. Catalyzed reporter deposition, a novel method of signal amplification. II. Application to membrane immunoassays. J Immunol Methods. 1991;137:103112.

27. Hopman AH, Ramaekers FC, Speel EJ. Rapid synthesis of biotin-, digoxigenin-, trinitrophenyl-, and fluorochrome-labeled tyramides and their application for in situ hybridization using CARD amplification. J Histochem Cytochem. 1998;46:771-777.

28. Rotstein NP, Aveldaño MI, Barrantes FJ, Politi LE. Docosahexaenoic acid is required for the survival of rat retinal photoreceptors in vitro. J Neurochem. 1996;66:1851-1859.

29. Politi LE, Bouzat C, de los Santos EB, Barrantes FJ. Heterologous retinal cultured neurons and cell adhesion molecules induce clustering of acetylcholine receptors and polynucleation in mouse muscle BC3H-1 clonal cell line. J Neurosci Res. 1996;43:639-651.

30. Adler R. Regulation of neurite growth in purified retina neuronal cultures: effects of PNPF, a substratum-bound, neurite-promoting factor. J Neurosci Res. 1982;8:165-177.

31. Insua MF, Garelli A, Rotstein NP, German OL, Arias A, Politi LE Cell cycle regulation in retinal progenitors by glia-derived neurotrophic factor and docosahexaenoic acid. Invest Ophthalmol Vis Sci. 2003;44:2235-2244.

32. Politi L, Rotstein N, Carri N. Effects of docosahexaenoic acid on retinal development: cellular and molecular aspects. Lipids. 2001; 36:927-935.

33. Politi LE, Rotstein NP, Carri NG. Effect of GDNF on neuroblast proliferation and photoreceptor survival: additive protection with docosahexaenoic acid. Invest Ophthalmol Vis Sci. 2001;42:30083015.

34. Barnstable CJ. Monoclonal antibodies which recognize different cell types in the rat retina. Nature. 1980;286:231-235.

35. Molday RS, Hicks D, Molday L. Peripherin: a rim-specific membrane protein of rod outer segment discs. Invest Ophthalmol Vis Sci. $1987 ; 28: 50-61$.

36. Uchihara T, Nakamura A, Nagaoka U, Yamazaki M, Mori O. Dual enhancement of double immunofluorescent signals by CARD: participation of ubiquitin during formation of neurofibrillary tangles. Histochem Cell Biol. 2000;114:447-451.

37. Laemmli UK, Beguin F, Gujer-Kellenberger G. A factor preventing the major head protein of bacteriophage $\mathrm{T} 4$ from random aggregation. J Mol Biol. 1970;47:69-85.

38. Tuohy G, Millington-Ward S, Kenna PF, Humphries P, Farrar GJ. Sensitivity of photoreceptor-derived cell line (661W) to baculoviral p35, Z-VAD.FMK, and Fas-associated death domain. Invest $O p h$ thalmol Vis Sci. 2002;43:3583-3589.

39. Slee EA, Zhu H, Chow SC, MacFarlane M, Nicholson DW, Cohen GM. Benzyloxycarbonyl-Val-Ala-Asp (OMe) fluoromethylketone (ZVAD.FMK) inhibits apoptosis by blocking the processing of CPP32. Biochem J. 1996;315:21-24.

40. Rotstein NP, Politi LE, Aveldaño MI. Docosahexaenoic acid promotes differentiation of developing photoreceptors in culture. Invest Ophthalmol Vis Sci. 1998;39:2750-2758.

41. Rotstein NP, Politi LE, German OL, Girotti R. Protective effect of docosahexaenoic acid on oxidative stress-induced apoptosis of retina photoreceptors. Invest Ophthalmol Vis Sci. 2003;44:22522259.
42. German OL, Insua MF, Gentili C, Rotstein NP, Politi LE. Docosahexaenoic acid prevents apoptosis of retina photoreceptors by activating the ERK/MAPK pathway. J Neurochem. 2006;98:15071520.

43. Chucair AJ, Rotstein NP, Sangiovanni JP, During A, Chew EY, Politi LE. Lutein and zeaxanthin protect photoreceptors from apoptosis induced by oxidative stress: relation with docosahexaenoic acid. Invest Ophthalmol Vis Sci. 2007;48:5168-5177.

44. Rotstein NP, Aveldaño MI, Barrantes FJ, Roccamo AM, Politi LE. Apoptosis of retinal photoreceptors during development in vitro: protective effect of docosahexaenoic acid. J Neurochem. 1997;69: $504-513$.

45. Hogback S, Leppimaki P, Rudnas B, Bjorklund S, Slotte JP, Tornquist $\mathrm{K}$. Ceramide 1-phosphate increases intracellular free calcium concentrations in thyroid FRTL-5 cells: evidence for an effect mediated by inositol 1,4,5-trisphosphate and intracellular sphingosine 1-phosphate. Biochem J. 2003;370:111-119.

46. Dasgupta U, Bamba T, Chiantia S, et al. Ceramide kinase regulates phospholipase $\mathrm{C}$ and phosphatidylinositol 4, 5, bisphosphate in phototransduction. Proc Natl Acad Sci U S A. 2009;106:2006320068.

47. Carter-Dawson LD, LaVail MM. Rods and cones in the mouse retina. II. Autoradiographic analysis of cell generation using tritiated thymidine. J Comp Neurol. 1979;188:263-272.

48. Morrow EM, Furukawa T, Cepko CL. Vertebrate photoreceptor cell development and disease. Trends Cell Biol. 1998;8:353-358.

49. Gómez-Muñoz A, Duffy PA, Martin A, et al. Short-chain ceramide1-phosphates are novel stimulators of DNA synthesis and cell division: antagonism by cell-permeable ceramides. Mol Pharmacol. 1995; $47: 833-839$.

50. Gangoiti P, Camacho L, Arana L, et al. Control of metabolism and signaling of simple bioactive sphingolipids: implications in disease. Prog Lipid Res. 2010;49:316-334.

51. Murakami M, Ito $H$, Hagiwara $K$, et al. ATRA inhibits ceramide kinase transcription in a human neuroblastoma cell line, SH-SY5Y cells: the role of COUP-TFI. J Neurochem. 2010;112:511-520.

52. Garelli A, Rotstein NP, Politi LE. Docosahexaenoic acid promotes photoreceptor differentiation without altering Crx expression. Invest Ophthalmol Vis Sci. 2006;47:3017-3027.

53. Pettus BJ, Bielawska A, Spiegel S, Roddy P, Hannun YA, Chalfant CE. Ceramide kinase mediates cytokine- and calcium ionophoreinduced arachidonic acid release. J Biol Chem. 2003;278:3820638213.

54. Mathias S, Dressler KA, Kolesnick RN. Characterization of a ceramide-activated protein kinase: stimulation by tumor necrosis factor alpha. Proc Natl Acad Sci U S A. 1991;88:10009-10013.

55. Mitra P, Maceyka M, Payne SG, et al. Ceramide kinase regulates growth and survival of A549 human lung adenocarcinoma cells FEBS Lett. 2007;581:735-740.

56. Gómez-Muñoz A, Kong JY, Parhar K, et al. Ceramide-1-phosphate promotes cell survival through activation of the phosphatidylinositol 3-kinase/protein kinase B pathway. FEBS Lett. 2005;579: 3744-3750.

57. Gómez-Muñoz A, Kong JY, Salh B, Steinbrecher UP. Ceramide-1phosphate blocks apoptosis through inhibition of acid sphingomyelinase in macrophages. J Lipid Res. 2004;45:99-105. 